\title{
Endovascular Therapy: The Second Round Begins
}

\author{
Jin Soo Lee, ${ }^{\mathrm{a}}$ Keun-Sik Hong ${ }^{\mathrm{b}}$ \\ aDepartment of Neurology, Ajou University School of Medicine, Suwon, Korea \\ ${ }^{b}$ Department of Neurology, Ilsan Paik Hospital, Inje University, Goyang, Korea
}

Recently, randomized controlled trials have consistently confirmed the benefit of endovascular therapy in patients with acute large intracranial artery occlusion up to 6 hours. With these results, major stroke guidelines recommend endovascular recanalization therapy as standard stroke care. Now that we are moving towards expansion of the therapeutic time window, better patient selection, expediting and enhancing recanalization, and the addition of neuroprotective agents are becoming necessary. As one of the major steps forward, the DAWN trial investigators presented exciting results that demonstrated the benefit of endovascular therapy up to 24 hours in patients with target mismatch at the 3rd European Stroke Organisation Conference 2017.

Despite technical advances in endovascular recanalization therapy, the HERMES collaboration study pooling individual patient-level data of 5 endovascular trials showed that 29\% of patients in the endovascular arm failed to achieve successful recanalization defined as modified Treatment in Cerebral Ischemia grade $2 b-3 .{ }^{1}$ The recanalization failures are mostly attributable to anatomical difficulties hindering access to and/or retrieving the thrombus, proximal tandem steno-occlusion, a resistant clot, and intracranial atherosclerosis-related re-occlusion. Neurointerventionalists often encounter these dilemmas, but their solutions have not been proven in randomized clinical trials.

In the current issue of Journal of Stroke, three review articles address these challenges in endovascular therapy. Yoo and Andersson discussed various obstacles and challenges in vascular access and thrombus removal, and provided a conceptual framework for better understanding the device-thrombus-vessel interaction. ${ }^{2}$ Kim summarized detailed thrombectomy techniques and illustrated challenging cases such as tandem occlusion, severe vascular tortuosity, huge or hard clots that are re- fractory to thrombectomy, and re-occlusion. ${ }^{3}$ Finally, Lee et al. focused on acute large intracranial atherosclerotic occlusion, ${ }_{1}^{4}$ which is more common in Asians than non-Asians, ${ }^{5}$ and requires more challenging techniques for successful endovascular therapy. They discussed clinical and imaging clues to differentiate acute intracranial atherosclerotic occlusion from embolic occlusion, which will help clinicians to establish an appropriate recanalization strategy.

The three articles also reviewed the recent technical refinements and therapeutic options to overcome the challenges. The suggested therapeutic approaches are largely anecdotal, based on observational studies or expert experiences. However, in the absence of trial-based evidence, it would be worthwhile to listen to these expert opinions when we encounter challenging cases. There is no controversy that successful reperfusion is the key to clinical benefit, and thereby we should develop more effective endovascular therapies. Now, the second round begins.

\section{References}

1. Goyal M, Menon BK, van Zwam WH, Dippel DW, Mitchell PJ, Demchuk AM, et al. Endovascular thrombectomy after largevessel ischaemic stroke: a meta-analysis of individual patient data from five randomised trials. Lancet 2016;387:17231731.

2. Yoo AJ, Andersson T. Thrombectomy in acute ischemic stroke: challenges to procedural success. J Stroke 2017;19:121-130.

3. Kim BM. Causes and solutions of endovascular treatment failure. J Stroke 2017;19:131-142.

4. Lee JS, Hong JM, Kim JS. Diagnostic and therapeutic strategies for acute intracranial atherosclerosis-related occlusions. J Stroke 2017;19:143-151.

5. Lee JS, Hong JM, Lee KS, Suh HI, Demchuk AM, Hwang YH, 
et al. Endovascular therapy of cerebral arterial occlusions: Intracranial atherosclerosis versus embolism. J Stroke Cerebrovasc Dis 2015;24:2074-2080.
Correspondence: Keun-Sik Hong

Department of Neurology, Ilsan Paik Hospital, Inje University, 170 Juhwa-ro, Ilsanseo-gu, Goyang 10380, Korea

Tel: +82-31-910-7680

Fax: +82-31-910-7368

E-mail: nrhks@paik.ac.kr

The authors have no financial conflicts of interest. 